\title{
Nonlinear MHD waves and discontinuities in the Martian magnetosheath. Observations and 2D bi-ion MHD simulations
}

\author{
Konrad Sauer $^{1,4}$, Eduard Dubinin ${ }^{1,2,4}$, and Klaus Baumgärtel ${ }^{3,4}$ \\ ${ }^{1}$ Max-Planck-Institut für Aeronomie D-37191 Katlenburg-Lindau, Germany \\ ${ }^{2}$ Space Research Institute, Russian Academy of Sciences, 117810 Moscow, Russia \\ ${ }^{3}$ Astrophysikalisches Institut D-14437 Potsdam, Germany \\ ${ }^{4}$ International Space Science Institute (ISSI), Hallerstrasse 6, 3012 Bern, Switzerland
}

(Received August 4, 1997; Revised January 19, 1998; Accepted May 19, 1998)

\begin{abstract}
The characteristic scale of the Martian magnetosheath is less than the pick-up gyroradius of oxygen ions. This leads to admissible differential motion of protons and heavies and a strong coupling between both ion fluids. 2D biion MHD simulations reveal many new interesting features in such Large Larmour Radius systems. The formation of an ion-composition boundary, which separates both plasmas, and structuring of the transition from proton dominated plasma of the solar wind origin to massive planetary plasma are the main features of the interaction. A comprehensive multi-instrument study of Martian plasma environment and the comparison with theoretical modelling initiated in the framework of the Visiting Science Programme of the International Space Science Institute (ISSI) in Bern (Switzerland) gives confirmation that Mars interacts with the solar wind like a comet which has a outgassing rate near to that of Grigg-Skjellerup. The results may also be relevant for small bodies which are surrounded by a neutral gas atmosphere (icy moons, asteroids, Mercury).
\end{abstract}

\section{Introduction}

The transition from the supersonic solar wind to the magnetospheric obstacle occurs via a sequence of standing MHD waves and discontinuities which accommodate all necessary changes in the plasma flow and the magnetic field. The bow shock, formed by fast magnetosound waves in front of an obstacle, is needed to divert the supersonic flow. The intermediate Alfven wave is required to provide a rotational transition from the draped interplanetary magnetic field to the magnetospheric field configuration. A slow MHD wave adjusts pressures outside and inside of the magnetosphere. Unmagnetized planets give us another example of the magnetosheath that is free from complications produced by the magnetic reconnection with an intrinsic planetary field. On the other hand, the addition of a new ion planetary population to a hydrogen magnetoplasma drastically modifies the dispersion of hydromagnetic waves and may produce new types of MHD discontinuities in the transition region. Recently, bi-ion MHD simulations (Sauer et al., 1996c) have demonstrated a bow shock "splitting" in bi-ion flows. A second discontinuity is formed downstream of the bow shock. It was assumed that the purpose of this "heavy ion discontinuity" (HID) is to provide a diversion of a heavy ion flow around the obstacle. To address the problem of HID's generation in more detail 2D bi-ion MHD simulations of the solar wind interaction with heavy ion sources were carried out. Application of these results to Mars is given.

Copy right (C) The Society of Geomagnetism and Earth, Planetary and Space Sciences (SGEPSS); The Seismological Society of Japan; The Volcanological Society of Japan; The Geodetic Society of Japan; The Japanese Society for Planetary Sciences.

\section{Observations}

The Phobos-2 observations near Mars provided some unexpected findings about the magnetospheric boundaries (Fig. 1). Boundaries were named on the basis of single instrument observations. Different terms were used to indicate changes of plasma and field characteristics during the transition from the shocked solar wind toward the magnetosphere. Recently, it was clarified that the magnetopause (MP), planetopause (PP), and ion composition boundary (ICB) are collocated and, probably, are the same (Dubinin et al., 1996a; Trotignon et al., 1996). This boundary separates the magnetosheath from the boundary layer/plasma mantle filled by slowly moving dense planetary plasma. The magnetosheath is mainly populated by protons although planetary ions are not restricted to the inner magnetosphere where they dominate. Another boundary called "mass-loading boundary (MLB)" (Lundin et al., 1991, 1993; Dubinin et al., 1997) which is located in the magnetosheath between the bow shock and ICB (MP, PP) remains a puzzle.

According to the definition of Lundin et al. (1991) the MLB marks the inner border of regular magnetosheath flow and outer limit of steady fluxes of planetary ions. Figure 2 clarifies the identification of boundaries. Figure 2 shows variations of $n\left(\mathrm{H}^{+}\right) / n\left(\mathrm{O}^{+}\right)$and $\mathrm{v}\left(\mathrm{O}^{+}\right) / \mathrm{v}\left(\mathrm{H}^{+}\right)$measured by the ASPERA instrument in the outbound path of the spacecraft on February 11, 1989. The spacecraft left the magnetosphere at about 17:00 UT and was sliding in the magnetosheath. The bow shock was crossed at 23:15 UT. In the outer magnetosheath $(>20: 30 \mathrm{UT})$, where the number density of $\mathrm{O}^{+}$ ions is small $(\sim 2-4 \%)$, the $\mathrm{v}_{x}$ component of the oxygen bulk velocity exceeds the local proton speed, although we can't exclude a certain overestimation of the bulk velocity of 


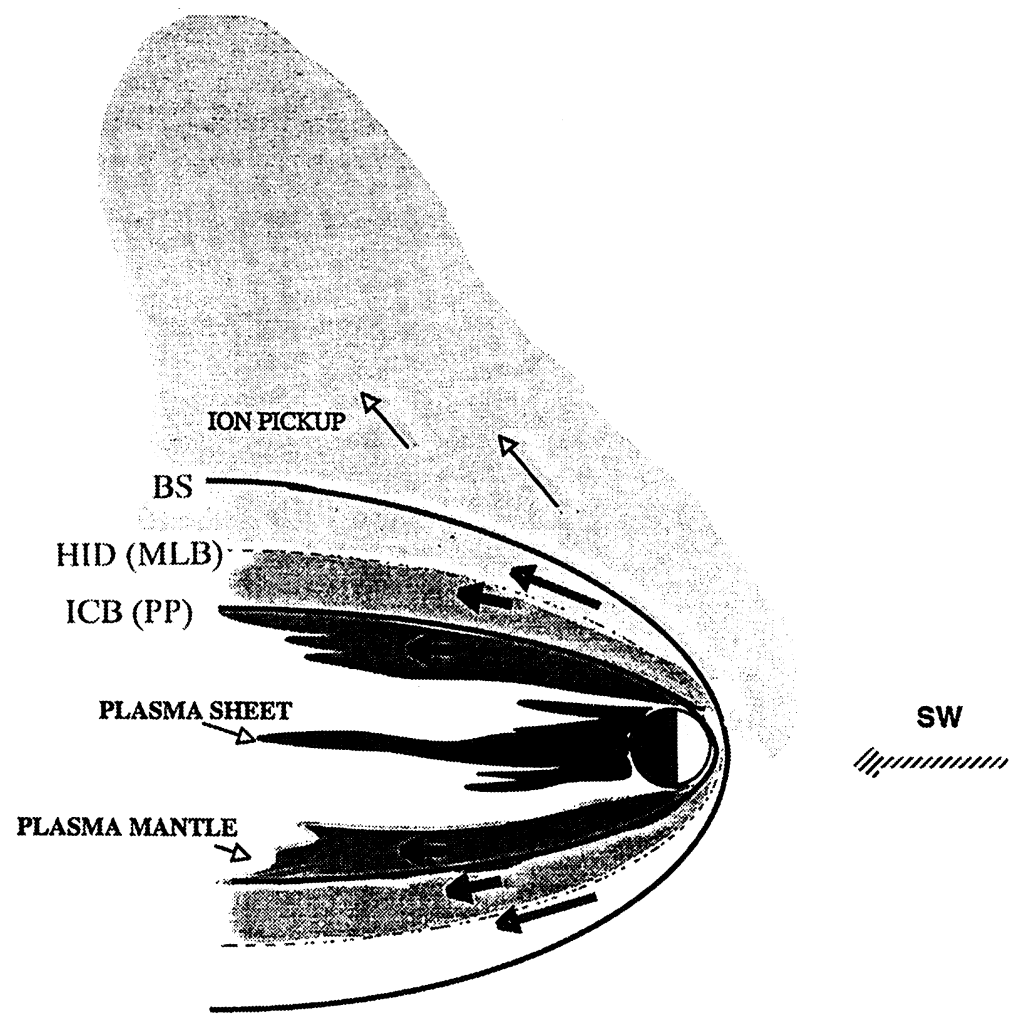

Fig. 1. Scheme with the main Martian magnetospheric boundaries-bow shock (BS), mass-loading boundary (MLB) or heavy ion discontinuity (HID), ion composition boundary (ICB) or planetopause (PP).

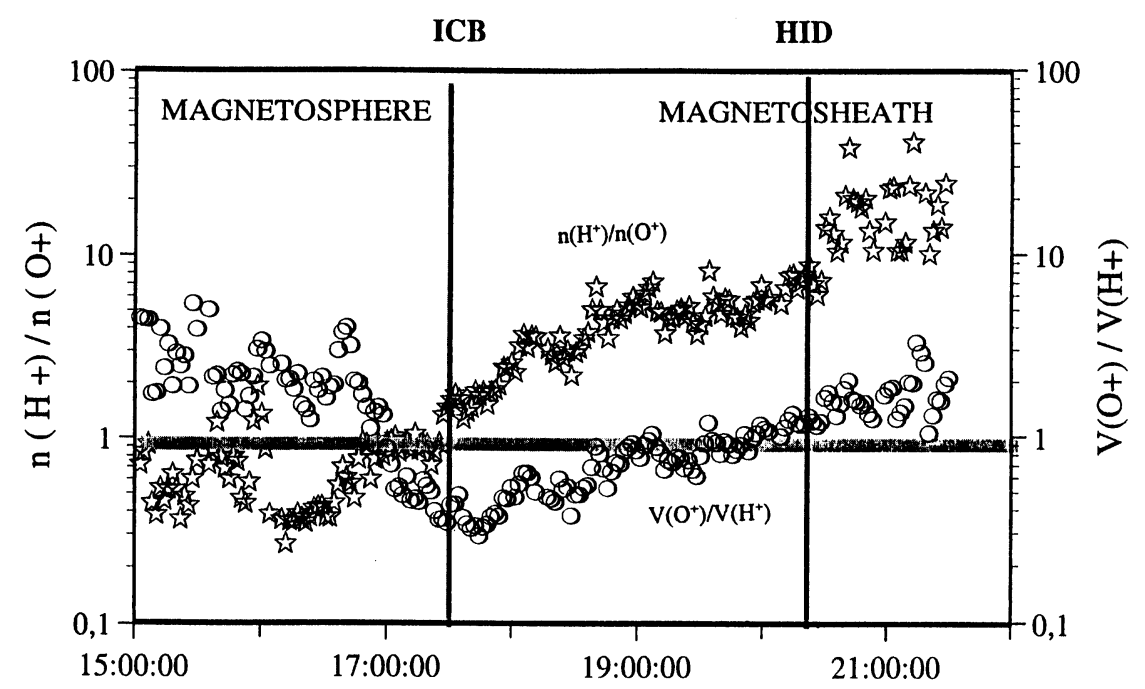

Fig. 2. Variations of $n\left(\mathrm{H}^{+}\right) / n\left(\mathrm{O}^{+}\right)$and $\mathrm{v}\left(\mathrm{O}^{+}\right) / \mathrm{v}\left(\mathrm{H}^{+}\right)$measured by the ASPERA instrument in the outbound part of the elliptical orbit on February 11, 1989. Positions of HID and ICB are shown by vertical lines.

heavies, evaluated from the onboard moment calculations, due to a falling of some protons into the oxygen channel. These "high speed" planetary ions were probably picked up by the solar wind upstream of the bow shock, and then, underwent a much smaller velocity jump at the bow shock as compared with that of protons. Therefore, the outer magnetosheath may be considered as a region where a "regime of test particles" is realized. A distinct transition is observed at $\sim 20: 30$ UT. The ratio of $n\left(\mathrm{H}^{+}\right) / n\left(\mathrm{O}^{+}\right)$drops at 2-3 times because of an increase of $n\left(\mathrm{O}^{+}\right)$. Then, both ion populations begin to flow with the same speed. It is interesting to note, that the transition occurs when the mass density of heavy ions becomes comparable with the mass density of shocked protons. In the central region of the magnetosheath, where 


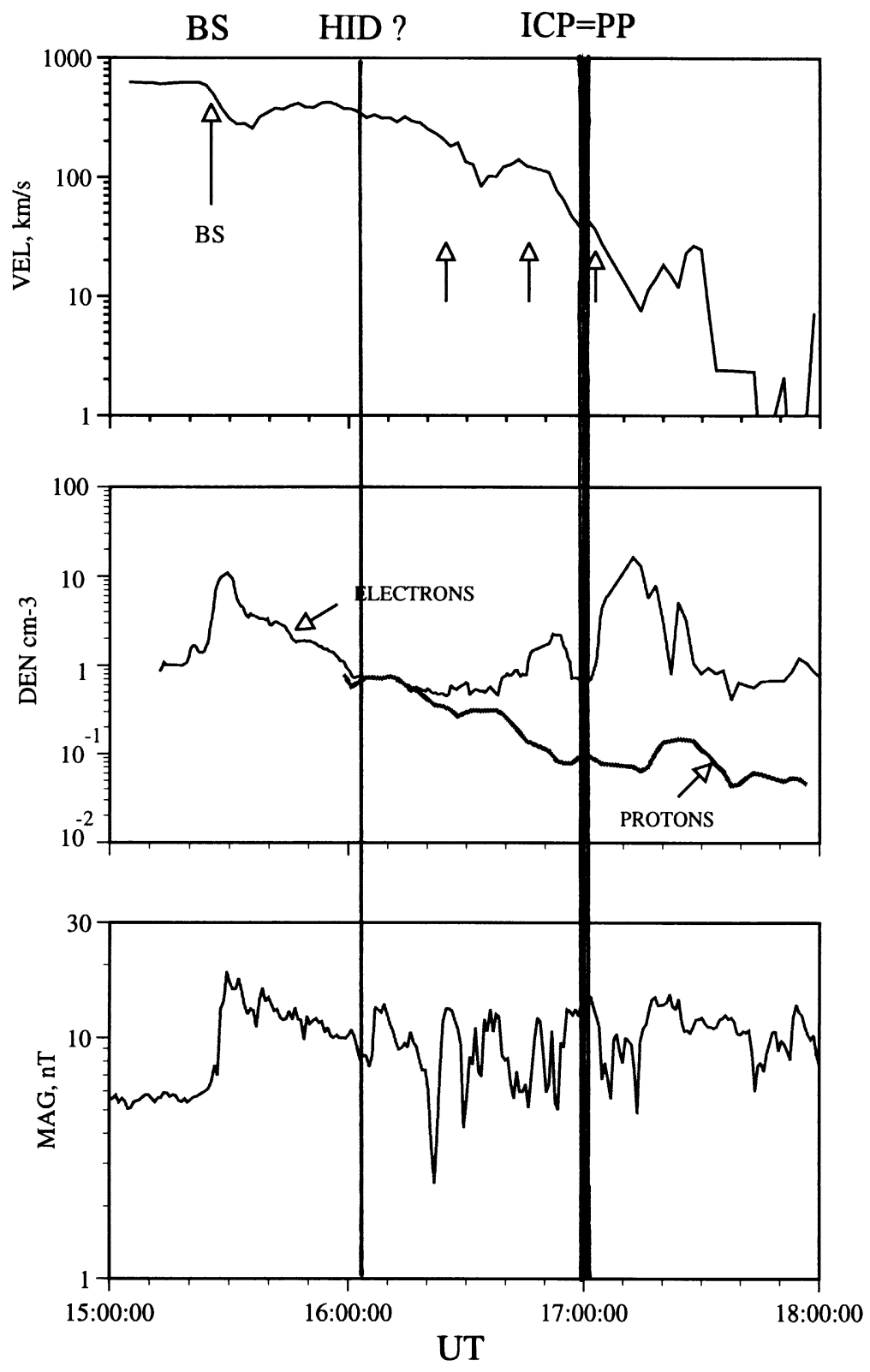

Fig. 3. Variation of the plasma parameters (proton velocity, electron and proton density, magnetic field) in the magnetosheath measured by Phobos-2 on March 2, 1989. A transition from proton dominated magnetosheath plasma to planetary plasma is seen from a splitting of the curves of the proton and electron number densities. A step-like deceleration of the solar wind is observed (upper panel). Tentative positions of HID and ICB are indicated by thin vertical lines.

the number density of heavies increases, protons outrun the oxygen flow. Lundin et al. (1991) and Lundin and Dubinin (1992) introduced the term "loaded regime" to emphasize pickup to low velocities. The process was discussed as internal loading of the MHD dynamo propelled by a driver solar wind plasma and resembles the momentum exchange between two colliding bodies. The important feature of the momentum exchange is that a differential streaming of both species in the central magnetosheath occurs in an oscillating manner indicating to the generation of bi-ion compressional waves. This wave-like momentum exchange between ion fluids is seen in the plot of the ratio $\mathrm{v}\left(\mathrm{O}^{+}\right) / \mathrm{v}\left(\mathrm{H}^{+}\right)$. A "divergence" of proton and heavy ion speeds is followed by their "convergence" $\left(\mathrm{v}\left(\mathrm{O}^{+}\right) / \mathrm{v}\left(\mathrm{H}^{+}\right) \rightarrow 1\right)$, emphasizing the ten- dency of the bi-ion streaming with equal velocities. Then, in the inner part of the magnetosheath, with an enlargement in the oxygen abundance, the differential speed of both species increases and a new transition boundary appears. This ion composition boundary is observed in the innermost region of the magnetosheath where the number density of planetary ions approaches the proton number density. The transition, accompanied by a sharp decrease of the proton bulk speed, occurs because planetary plasma becomes very dense and takes the largest fraction of the momentum from the shocked solar wind ("regime of overloading").

Observations in the Martian magnetosheath give also examples for strong bi-ion MHD waves evolving to multiple shocks. Figure 3 shows an example of variations of plasma 

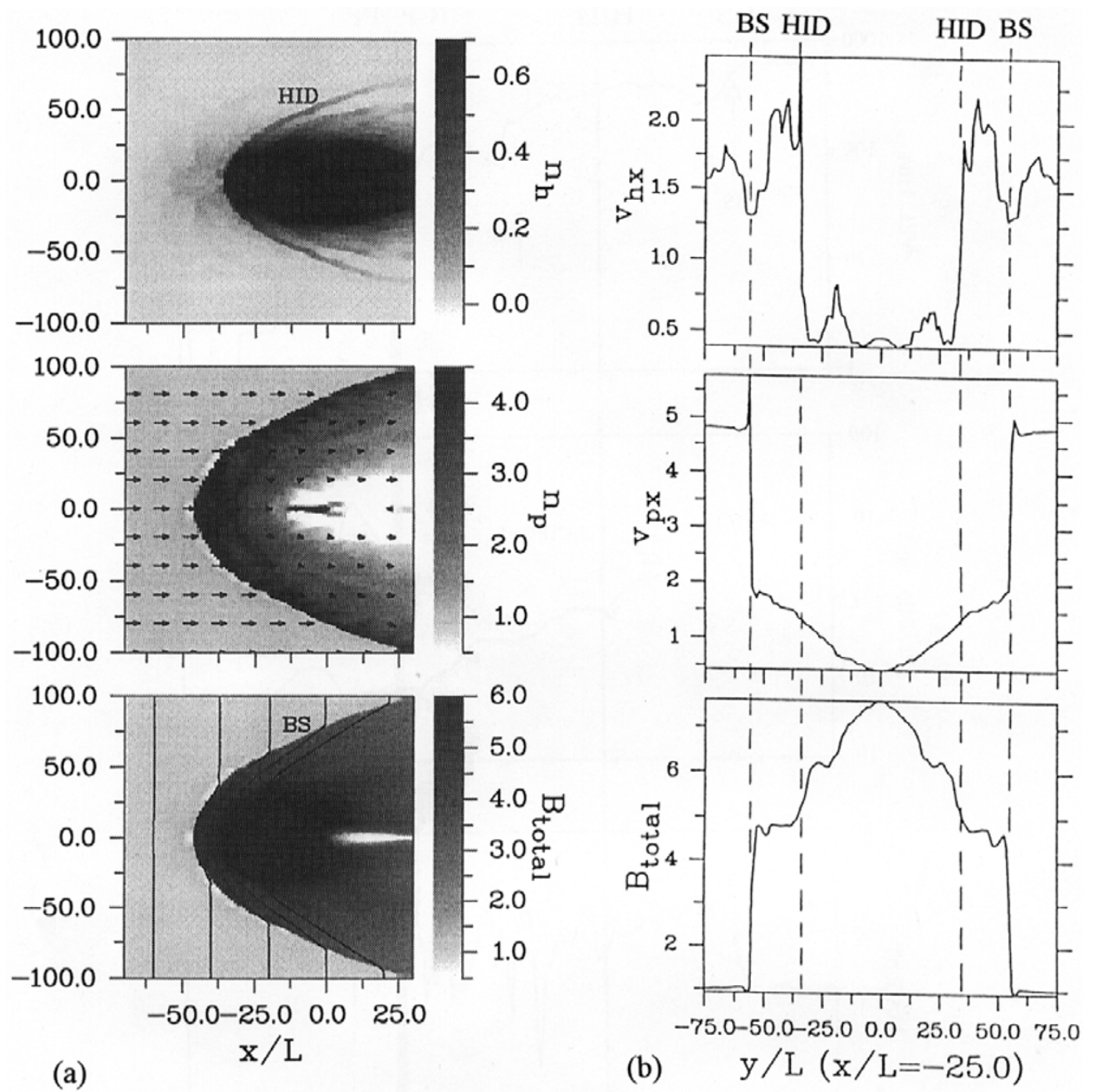

Fig. 4. 2D distribution of the density of heavies and protons from bi-ion fluid simulations. The bottom panel gives the magnetic field strength. The right column contains the cut made along the $y$-axis $(x / L=-25)$. The positions of the discontinuities (BS, HID) are marked. The velocities are normalized to the Alfven velocity. $L$ is the proton skin length.

parameters in the magnetosheath. A transition from proton dominated magnetosheath plasma to planetary plasma is clearly seen from a splitting of curves of proton and electron number densities. A step-like deceleration of the solar wind is observed (upper panel). Magnetic field measurements which were made with a better temporal resolution show the presence of strong nonlinear waves in this region. Another example of multiple shock-like structures is given in the accompanying paper of Dubinin et al. (1998).

\section{2D Bi-ion MHD Simulations}

In this paper we focus on the magnetosheath structure addressing the question whether the MLB is a real boundary or a smooth transition from a "classical pickup" to a "loaded pickup" mode occurs. To study a generation of a new discontinuity downstream the "proton bow shock", when the solar wind is loaded by a secondary ion population, the $2 \mathrm{D}$ collisionless bi-ion fluid model which treats the protons and heavy ions as separate fluids communicating each other only by means of electromagnetic forces was used. The solar wind interaction process at Mars is characterized by a direct streaming of the solar wind through an extended neutral corona (hot oxygen exosphere). Another population of planetary ions forms an ionospheric obstacle. In contrast to the Venus case, the Martian ionosphere is rather degenerated and lies mainly below the interaction region. The important feature of the Martian magnetosphere is that a characteristic scale of the magnetosheath is comparable with the proton gyroradius and less then the pickup $\mathrm{O}^{+}$gyroradius. In such systems the relative streaming of different ion species is admissible and bi-ion modified MHD gives a reasonable approach for the description of the gross dynamics of the system (Harold and Hassam, 1994; Sauer et al., 1994, 1995).

For Mars, a superposition of two exponential profiles with different scale lengths $h_{1,2}\left(h_{1}=2 L, h_{2}=10 L\right.$, where $L$ is the proton inertial length, $\left.L=\mathrm{v}_{\mathrm{A}} / \Omega_{\mathrm{p}}=c / \omega_{\mathrm{p}}\right)$ was taken to model both the thermal ionosphere and hot exosphere (Ip, 1988). The typical value of $L$ for the Martian environment is about of $100 \mathrm{~km}$. The strength $q_{\mathrm{h}}=n_{\mathrm{o}} v_{\mathrm{ph}}$ of the heavy ion source was chosen $q_{1}=0.1 \mathrm{~cm}^{-3} \mathrm{~s}^{-1}$ and 
$q_{2}=0.01 \mathrm{~cm}^{-3} \mathrm{~s}^{-1}$, where $n_{\mathrm{o}}$ is the neutral gas density and $v_{\mathrm{ph}}$ is the photoionization rate $\left(q_{\mathrm{h}}=q_{1} \exp \left(-r / h_{1}\right)+\right.$ $q_{2} \exp \left(-r / h_{2}\right)$. Since the bi-ion fluid model has been described in earlier papers (Sauer et al., 1994, 1995, 1997), we present here only basic equations.

Continuity and momentum equation for the solar wind protons

$$
\begin{gathered}
\frac{\partial}{\partial t} n_{\mathrm{p}}+\nabla \cdot\left(n_{\mathrm{p}} \mathbf{v}_{\mathrm{p}}\right)=0, \\
\frac{\partial}{\partial t}\left(n_{\mathrm{p}} \mathbf{v}_{\mathrm{p}}\right)+\nabla \cdot\left(n_{\mathrm{p}} \mathbf{v}_{\mathrm{p}} \mathbf{v}_{\mathrm{p}}+P_{\mathrm{p}}\right) \\
=\frac{1}{m_{\mathrm{p}}} \frac{n_{\mathrm{p}}}{n_{\mathrm{e}}}\left[e n_{\mathrm{c}} \mathbf{v}_{\mathrm{p}} \times \mathbf{B}+e n_{\mathrm{h}}\left(\mathbf{v}_{\mathrm{p}}-\mathbf{v}_{\mathrm{h}}\right) \times \mathbf{B}\right. \\
\left.-\nabla\left(\left(P_{\mathrm{e}}+\frac{B^{2}}{2 \mu_{0}}\right) I-\frac{\mathbf{B B}}{\mu_{0}}\right)\right],
\end{gathered}
$$

and for the heavy ions

$$
\begin{gathered}
\frac{\partial}{\partial t} n_{\mathrm{h}}+\nabla \cdot\left(n_{\mathrm{h}} \mathbf{v}_{\mathrm{h}}\right)=q_{\mathrm{h}}, \\
\frac{\partial}{\partial t}\left(n_{\mathrm{h}} \mathbf{v}_{\mathrm{h}}\right)+\nabla \cdot\left(n_{\mathrm{h}} \mathbf{v}_{\mathrm{h}} \mathbf{v}_{\mathrm{h}}+P_{\mathrm{h}}\right) \\
=\frac{1}{m_{\mathrm{h}}} \frac{n_{\mathrm{h}}}{n_{\mathrm{e}}}\left[e n_{\mathrm{p}}\left(\mathbf{v}_{\mathrm{h}}-\mathbf{v}_{\mathrm{p}}\right) \times \mathbf{B}\right. \\
\left.-\nabla\left(\left(P_{\mathrm{e}}+\frac{B^{2}}{2 \mu_{0}}\right) I-\frac{\mathbf{B B}}{\mu_{0}}\right)\right] .
\end{gathered}
$$

The above equations are closed with Faraday's law

$$
\frac{\partial \mathbf{B}}{\partial t}-\nabla \times\left[\frac{1}{n_{\mathrm{e}}}\left(n_{\mathrm{p}} \mathbf{v}_{\mathrm{p}}+n_{\mathrm{h}} \mathbf{v}_{\mathrm{h}}\right) \times \mathbf{B}\right]-\frac{\mathbf{B} \cdot \nabla \mathbf{B}}{\mu_{0} n_{\mathrm{e}}}=0 .
$$

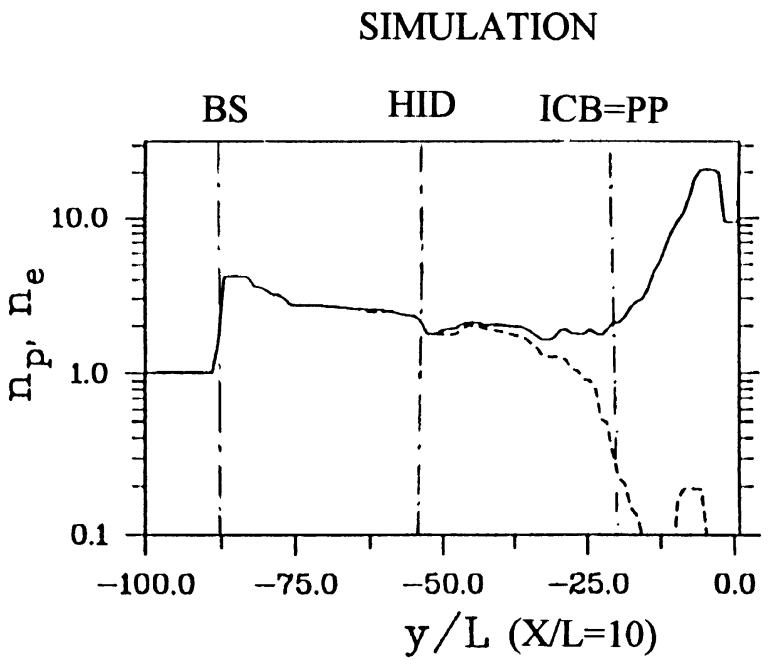

Fig. 5. Cut through the box in Fig. 4 at $x / L=10$ : The panel shows a gradual displacement of proton dominated plasma by planetary plasma from bi-ion modelling. The positions of HID and ICB = PP (PP stands here for protonopause indicating the terminal boundary of the proton flow, according to Sauer et al., 1994, 1995).
The electron pressure $P_{\mathrm{e}}=n_{\mathrm{e}} k T_{\mathrm{e}}$ is calculated for isothermal conditions $\left(T_{\mathrm{e}}=\right.$ const $)$, where the electron density follows from the quasi-neutrality condition, $n_{\mathrm{e}}=n_{\mathrm{p}}+n_{\mathrm{h}}$.

Figure 4 gives the 2D distribution of the heavy ion density $n_{\mathrm{h}}$, the proton number density $n_{\mathrm{p}}$ and the magnetic field strength $B_{\text {total }}$ as result of the interaction of the solar wind with the Martian exosphere/ionosphere. The Alfven Mach number of the incoming flow is $M_{\mathrm{A}}=5$. After $\Omega_{\mathrm{p}} t \approx 200$ a nearly steady state is reached. A distinct, parabolic bow shock is formed around the obstacle. A proton cavity in the central part of the interaction region appears, where heavy ion density increases $\left(n_{\mathrm{h}} / n_{\mathrm{p}} \geq 10\right)$. Figure 5 shows a splitting of the curves of proton and electron density, which indicates the transition from a proton dominated plasma to planetary plasma. Distances and velocities in these figures are respectively normalized to the ion skin length $L=c / \omega_{\mathrm{p}}$ and to the Alfven velocity of the proton fluid. Other parameters are normalized to their upstream values. The comparison with observations (Fig. 3) made near Mars gives a reasonable agreement. The important feature is that the process of replacement of protons by planetary ions is not a smooth, but is accompanied with the formation of several ray-like structures, well displayed in the Plate of the $n_{\mathrm{h}}$-distribution (Fig. 4). The behavior of both ion species is given in Fig. 4(b) which shows the cut made along the $y$ axis $(x=-25 L)$. A remarkable effect is a different behavior of ion populations at the bow shock. Whereas protons are decelerated to subAlfven velocities, the heavies are accelerated. Then, in the
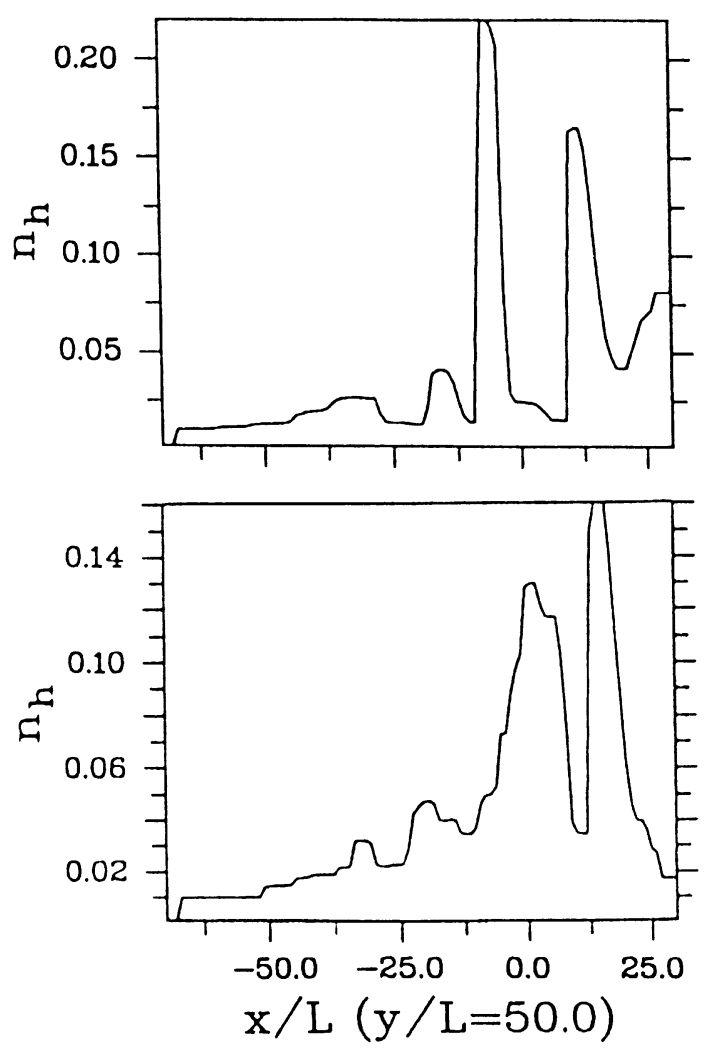

Fig. 6. Distribution of the heavy ion density along the $x$-axis $(y / L=50)$ at two subsequent moments of time $\left(\Delta t=\Omega_{i}^{-1}\right)$. Structures are travelling downstream. 

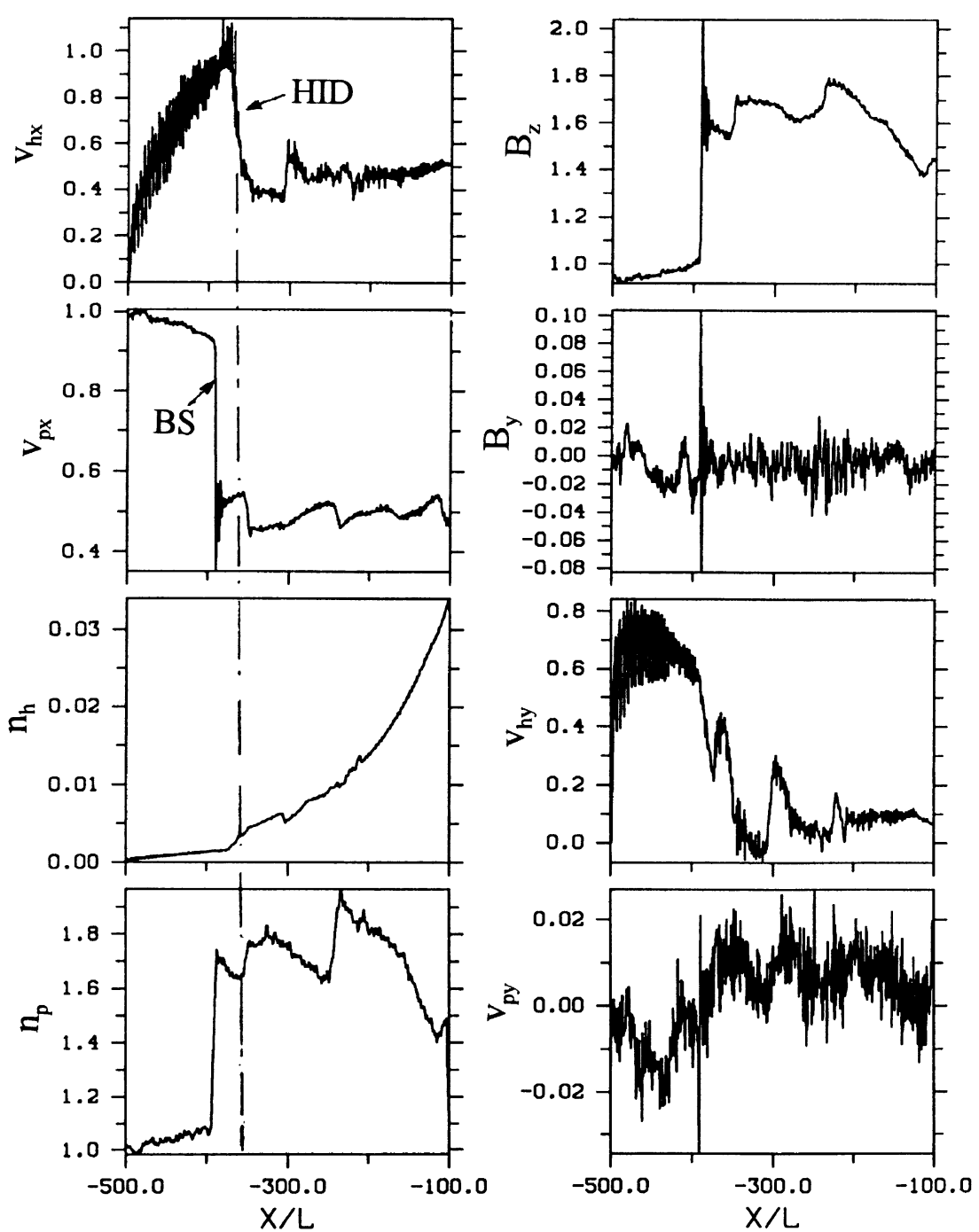

Fig. 7. Spatial separation between the proton shock (BS) and the heavy ion discontinuity (HID): Results of 1D hybrid code simulations of solar wind interaction with a cometary source (like Grigg-Skjellerup). The undisturbed magnetic field is in the $z$ direction. Different from Fig. 4, the proton parameters and the magnetic field are normalized to their undisturbed values. The Mach number is $M_{\mathrm{A}}=5$.

magnetosheath, another sharp transition is observed (Heavy Ion Discontinuity = HID). The decrease of velocity of heavies is accompanied by an enhancement of their number density and a distinct change of the magnetic field. According to our present understanding, the HID results from the large relative velocity inside the proton shock (BS) between the shocked protons and the flow of heavy ions which (due to their larger mass) undergo only a weak deceleration by the BS associated electric field (e.g. Motschmann et al., 1991). As a consequence, a strong Lorentz force arises which leads to deceleration and subsequent "shock" formation in the heavy ion flow. Inside the HID, protons and heavies flow with nearly the same velocity. The appearance of new substructures inside the HID $(y / L \sim \pm 20)$ is also seen.

Another interesting feature is the nonstationary behavior of the observed rays. Figure 6 shows the distribution of the heavy ion number density along the $x$-axis $(y / L=50)$ at subsequent moments of time. Structures resemble nonlinear MHD waves carried downstream by the plasma flow. This indicates a dynamic process rather than a steady state pattern.
Structures are moving away and new ones arise again. Thus, Fig. 4 should be thought of a snapshot picture of the interaction process. To demonstrate unambiguously the appearance of a "heavy ion" discontinuity downstream the proton bow shock, Sauer et al. (1996c) have considered (instead of solar wind massloading) the case of a pregiven bi-ion plasma flow which interacts with an immobile ion cloud as the obstacle. Whereas the protons are slowed down to sub-Alfven velocities, the heavies move with the undisturbed speed before they undergo their shock-like transition. At this transition the heavy ions are strongly deflected and bunched. Their flow occurs in a narrow channel around the obstacle. As discussed above, the abrupt deceleration of the heavy ion flow is believed to be caused by the Lorenz force which is associated with a differential streaming of both ion flows. From these studies, it is also concluded that multiple splitting, which is clearly observed in 2D bi-ion MHD simulations under massloading conditions, arises due to the continuous production of heavy ions and the repeated occurrence of differential streaming. Finally, it should be mentioned that HID formation was 

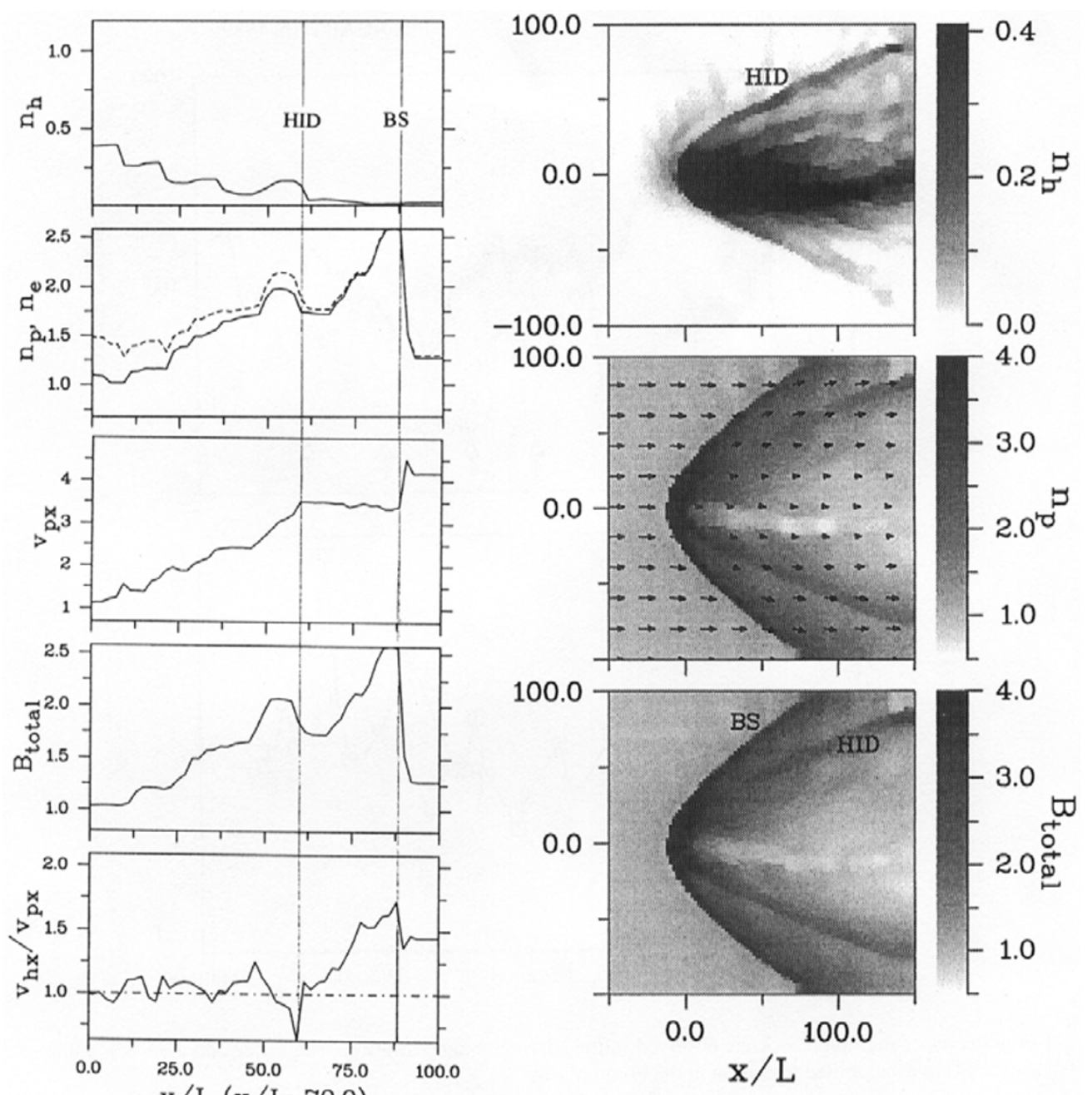

Fig. 8. Right column gives a spatial distribution of the heavy ion density, the proton number density and the magnetic field strength realized in the $2 \mathrm{D}$ bi-ion MHD simulation of the solar wind interaction with a cometary source. Different from Fig. 7, the proton velocity is normalized by the Alfven velocity. The bow shock (BS) and the heavy ion discontinuity (HID) are well seen. Multiple structures inside the HID arise due to a continuous production of new heavy ions and a repeated occurrence of differential streaming. The left column contains the $y$ variation $(x / L=70)$ of $n_{\mathrm{h}}, n_{\mathrm{p}}$ (dashed curve) and $n_{\mathrm{e}}$ (solid curve); the velocity of protons $\left(\mathrm{v}_{\mathrm{px}}\right)$ and the magnetic field strength $\left(B_{\mathrm{total}}\right)$. The bottom panel shows the ratio $\mathrm{v}_{\mathrm{hx}} / \mathrm{v}_{\mathrm{px}}$. Oscillations in the momentum exchange between protons and heavies are observed.

found as a characteristic feature in relevant kinetic hybrid code simulations. An example is given in Fig. 7.

A periodicity in the process of the momentum exchange between protons and heavies is also revealed in $2 \mathrm{D}$ bi-ion MHD simulations. The plate of Fig. 8 depicts the distribution of the heavy ion density $n_{\mathrm{h}}$, the proton density $n_{\mathrm{p}}$ and the magnetic field strength. The undisturbed magnetic field was transverse to the simulation plane. Although the $1 / r^{2}$ dependence of the source power, which is more appropriate to the comet case, was used, the same features of the interaction remain. The discontinuity (HID) downstream of the bow shock, formed in the heavy ion flow, is clearly observed in the distribution of the proton number density and the magnetic field strength. The left column contains the cut through the simulation box along the $y$-axis $(x / L=70)$. The position of the bow shock (BS) and the HID are marked by thin lines. The bottom panel gives the ratio of bulk speeds of heavies and protons. In the outer magnetosheath, the speed of heavies exceeds the proton speed. Then, near the HID, the relative streaming of both ion species occurs in a periodical manner with a decreasing amplitude of oscillations. Such behavior of protons and heavies is similar to that was observed by the Phobos-2 in the Martian magnetosheath (Fig. 2). The important difference, as compared with Mars and the previous simulation run (Fig. 4) is an absence of the magnetic field draping in the given 2D geometry (the magnetic field is oriented perpendicular to the simulation plane). In the real 3D configuration and in the simulations shown in Fig. 4, where the magnetic field was in the $x y$-plane, draping is provided by the transfer of some fraction of the momentum from the shocked solar wind to the magnetic field, e.g. via a generation of Alfven wings, and heavies are able to take less fraction of the momentum from protons than for the case without draping. This leads to a gradual "splitting" of both flows and an increase of the relative speed between ion species. Heavies gain less velocity and protons begin to outrun heavies. Measurements in the central Martian magnetosheath, where the draping of the magnetic field occurs and oxygen abundance 

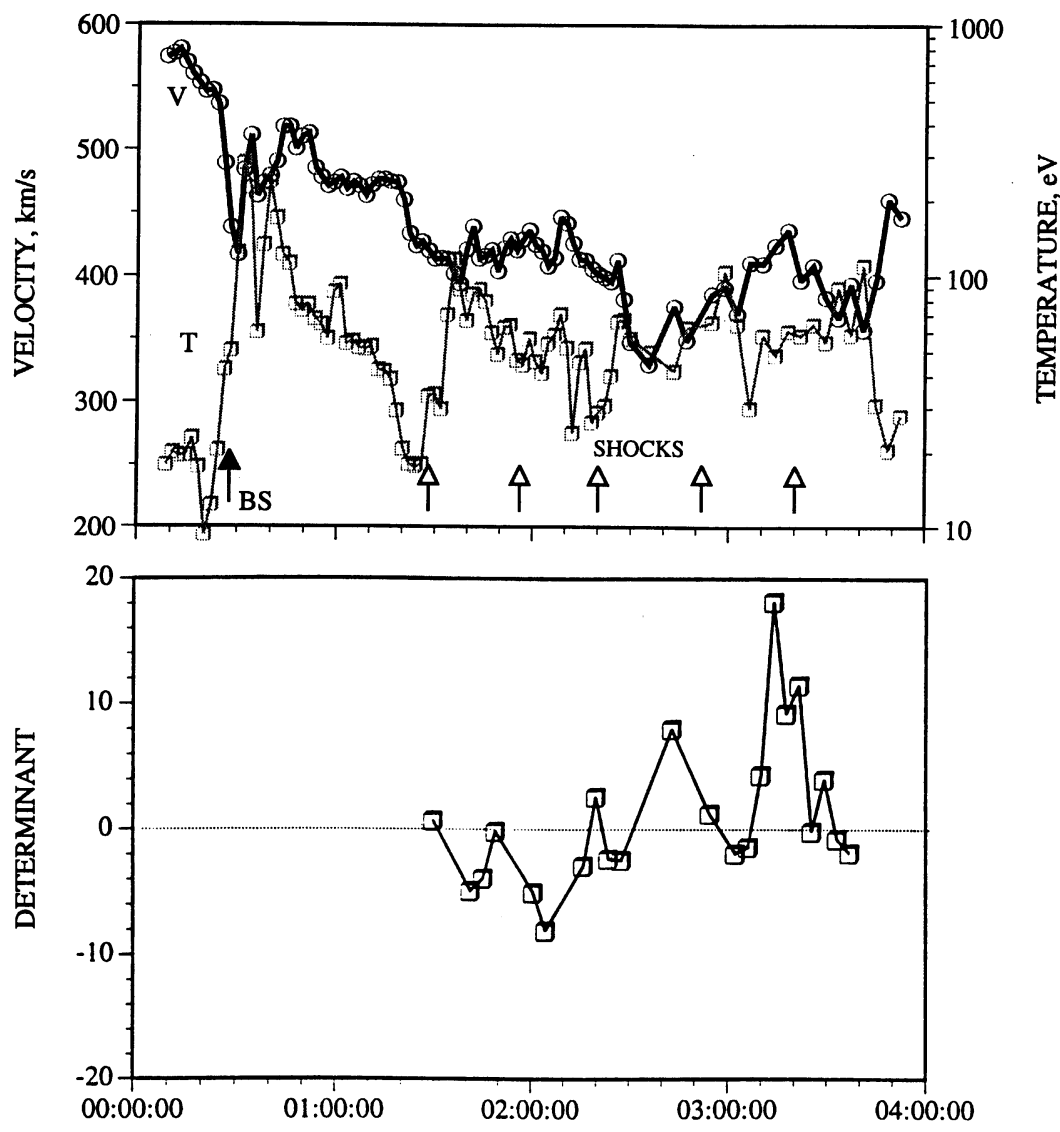

Fig. 9. Upper panel shows a set of multiple shocklets observed in the Martian magnetosheath. The lower one gives the value of determinant $\Delta$. The condition $\Delta=0$ corresponds to a shock-like transition in the bi-ion plasma.

increases, confirms this scenario (Fig. 2).

This coupling in a multi-ion plasma leads to the appearance of additional compressive wave modes and prompts a question about multi-ion shocks. Indeed, Dubinin et al. (1996b, 1997) have observed multiple shocklets in the Martian transition region. Similar stratification of plasma on alternating strings with bunched flows of heavies is distinctly observed in the 2D bi-ion MHD simulations. The idea of new singular points in bi-ion plasma is natural and was already addressed (McKenzie et al., 1993). In contrast to the usual proton-electron plasma in which this singularity occurs when the proton bulk speed matches the "sound" speed, in a bi-ion plasma critical or singularity points degenerate to sound speeds of different ion species only in the extreme cases when $n_{\mathrm{p}} \gg n_{\mathrm{h}}$ or $n_{\mathrm{h}} \gg n_{\mathrm{p}}$. In the general case of arbitrary abundance ratio, the motion of all three populations (electrons, protons and heavies) becomes strongly coupled and the singularity appears not for certain values of proton or heavy ion bulk speeds but rather when their combination lies in particular wave loci emphasizing the coupled nature of biion flow. The wave loci for the stationary one-dimensional problem is determined from the condition that a determinant $\Delta$ of the system of Eqs. (1)-(5) that describes spatial variations of plasma parameters equals to zero (McKenzie et al., 1993). The problem is intimately linked with the conditions under which stationary waves in bi-ion plasma may exist in a laboratory frame relative to which protons and heavy ions stream with different velocities. Considering the simplest case of magnetized electrons and unmagnetized protons and heavy ions (electrostatic approximation) which implies the neglect of the $\left(\mathbf{v}_{\mathrm{p}}-\mathbf{v}_{\mathrm{h}}\right) \times \mathbf{B}$ terms in the momentum equations (2) and (4), one gets the following dispersion relation for bi-ion magnetoacoustic waves (Sauer et al., 1996a,b):

$$
\left[\left(\mathrm{v}_{\mathrm{ph}}-\mathrm{v}_{\mathrm{p}}\right)^{2}-c_{\mathrm{Mp}}^{2}\right] \cdot\left[\left(\mathrm{v}_{\mathrm{ph}}-\mathrm{v}_{\mathrm{h}}\right)^{2}-c_{\mathrm{Mh}}^{2}\right]=c_{\mathrm{Mp}}^{2} c_{\mathrm{Mh}}^{2}
$$

where $\mathrm{v}_{\mathrm{ph}}=\omega / k$ is the phase velocity, $\mathrm{v}_{\mathrm{p}}$ and $\mathrm{v}_{\mathrm{h}}$ are bulk speeds of protons and heavies, and

$$
\begin{gathered}
c_{\mathrm{Mp}}=\left[\mathrm{v}_{\mathrm{Ap}}^{2} \frac{n_{\mathrm{p}}^{2}}{n_{\mathrm{e}}^{2}}+c_{\mathrm{sp}}^{2} \frac{n_{\mathrm{p}}}{n_{\mathrm{e}}}\right]^{1 / 2}, \\
c_{\mathrm{Mh}}=\left[\mathrm{v}_{\mathrm{Ah}}^{2} \frac{n_{\mathrm{h}}^{2}}{n_{\mathrm{e}}^{2}}+c_{\mathrm{sh}}^{2} \frac{n_{\mathrm{h}}}{n_{\mathrm{e}}}\right]^{1 / 2}, \\
c_{\mathrm{sp}}=\left(\frac{k T_{\mathrm{e}}}{m_{\mathrm{p}}}\right)^{1 / 2}, \quad c_{\mathrm{sh}}=\left(\frac{k T_{\mathrm{e}}}{m_{\mathrm{h}}}\right)^{1 / 2}, \\
\mathrm{v}_{\mathrm{Ap}}=\frac{B}{\left(\mu_{0} n_{\mathrm{p}} m_{\mathrm{p}}\right)^{1 / 2}, \quad \mathrm{v}_{\mathrm{Ah}}=\frac{B}{\left(\mu_{0} n_{\mathrm{h}} m_{\mathrm{h}}\right)^{1 / 2}}}
\end{gathered}
$$

are magnetosound, sound and Alfven speeds for protons and heavies in a bi-ion plasma. (The excitation of low-frequency 
electromagnetic waves by an unmagnetized beam of heavy ions in a plasma of magnetized electrons and protons is a topic of separate papers by Baumgärtel et al. (1998) and Sauer et al. (1998).)

Taking in Eq. (6) $\mathrm{v}_{\mathrm{ph}}=0$, we have

$$
\Delta=\left[\mathrm{v}_{\mathrm{p}}^{2}-c_{\mathrm{Mp}}^{2}\right] \cdot\left[\mathrm{v}_{\mathrm{h}}^{2}-c_{\mathrm{Mh}}^{2}\right]-c_{\mathrm{Mp}}^{2} c_{\mathrm{Mh}}^{2} .
$$

The condition $\Delta=0$ determines a shock-like transition in a bi-ion plasma when stationary spatially continuous massloading is no longer possible. The bottom panel of Fig. 9 shows the value of $\Delta$ evaluated from the plasma and the magnetic field measurements made by the ASPERA and the MAGMA instruments. Only "spectral" data of the ASPERA were used to evaluate the parameters of $\mathrm{H}^{+}$and $\mathrm{O}^{+}$ions. This procedure narrows the time interval of acceptable data compared with onboard moment data, but permits to avoid difficulties arising from a background level of the oxygen channel (Dubinin et al., 1996a). Positive values of the determinant $\Delta$ correspond to a "supersonic" flow. The position of shocks indicated by arrows in the upper panel of Fig. 9 is well recognized while the condition $\Delta=0$ is met.

\section{Summary}

1. The Martian magnetosheath is filled with compressional MHD waves. An oscillating character of the momentum exchange between protons and heavies is observed. A periodicity of the process is caused by the action of Lorentz force, related with a differential streaming of ion flows $(\sim \Delta \mathbf{v} \times \mathbf{B})$, and pressure forces which either accelerates/ decelerates heavies/protons or decelerates/accelerates heavies/protons. Observations in the Martian magnetosheath give also examples for nonlinear bi-ion MHD waves evolving to multiple shocks. Transition boundaries were observed in the magnetosheath. The intermediate transition, which marks the outer limit of steady flows of planetary ions, is considered as the heavy ion discontinuity (HID) whose purpose is to accommodate all required changes in heavy ion flow around the magnetosphere.

2. $2 \mathrm{D}$ bi-ion simulations show the existence of the intermediate transition. This plasma boundary, called the HID, resembles a shock-like transition for the heavy ion flow. A bunching of heavy ion flow due to a differential motion of ions is accompanied by a resonant excitation of multiple Machlike cones around the obstacle. This means that the transition from the shocked solar wind dominated by protons to planetary plasma occurs via a set of shock-like discontinuities. These structures could be interpreted as multiple HIDs generated due to continuous production of newly created heavy ions closer to the planetary/cometary obstacle and a repeated differential ion streaming. As a result, the heavy ion flow is deflected and bunched forming ray-like structures, often observed near comets.

Although the presented results must be considered as preliminary and a lot of unsolved questions remain, we hope that they help in understanding of the origin of intermediate transitions in the magnetosheath/cometosheath of nonmagnetized planets/comets.

Acknowledgments. The authors wish to express thanks to the ISSI in Bern for the support in the collaboration in the framework of Visiting Science Programme.

\section{References}

Baumgärtel, K., K. Sauer, E. Dubinin, V. Tarasov, and M. Dougherty, "Phobos events"- -signatures of solar wind interaction with a gas torus?, Earth Planets Space, 50, 453-462, 1998.

Dubinin, E., K. Sauer, R. Lundin, K. Baumgärtel, and A. Bogdanov, Structuring of the transition region (plasma mantle) of the Martian magnetosphere, Geophys. Res. Lett., 23, 785, 1996a.

Dubinin, E., K. Sauer, R. Lundin, O. Norberg et al., Plasma characteristics of the boundary layer in the Martian magnetosphere, J. Geophys. Res. 101, 27061-27075, 1996b.

Dubinin, E., K. Sauer, K. Baumgärtel, and R. Lundin, The Martian magnetosheath. Phobos-2 observations, Adv. Space Res., 20, (2)149-153, 1997.

Dubinin, E., K. Sauer, K. Baumgärtel, and K. Srivastava, Multiple shocks near Mars, Earth Planets Space, 50, 279-287, 1998.

Harold, J. B. and A. B. Hassam, Two ion fluid numerical investigations of solar wind gas releases, J. Geophys. Res., 99, 19325-19340, 1994.

Ip, W.-H., Exosphere of Mars, on hot oxygen corona of Mars, Icarus, 76, 135-145, 1988.

Lundin, R. and E. Dubinin, Phobos-2 results on the ionospheric plasma escape from Mars, Adv. Space Res., 12, (9)255-(9)263, 1992.

Lundin, R., E. Dubinin, H. Koskinen, O. Norberg, N. Pissarenko, and S. Barabash, On the momentum transfer of the solar wind to the Martian topside ionosphere, Geophys. Res. Lett., 18, 1059-1062, 1991.

Lundin, R., E. Dubinin, S. Barabash, and O. Norberg, ASPERA observations of Martian magnetospheric boundaries, in Plasma Environment of Nonmagnetic Planets, COSPAR colloq. Ser., v. 4, edited by T. Gombosi, pp. 311-320, Pergamon, New York, 1993.

McKenzie, J. F., E. Marsch, K. Baumgärtel, and K. Sauer, Wave and stability properties of multi-ion plasmas with applications to winds and flows, Ann. Geophys., 11, 341-353, 1993.

Motschmann, U., K. Sauer, T. Roatsch, and J. F. McKenzie, Subcritical multiple-ion shocks, J. Geophys. Res., 96, 13,841-13,848, 1991.

Sauer, K., A. Bogdanov, and K. Baumgärtel, Evidence of an ion composition boundary (protonopause) in bi-ion fluid simulations of solar wind massloading, Geophys. Res. Lett., 21, 2255, 1994.

Sauer, K., A. Bogdanov, and K. Baumgärtel, The protonopause-an ion composition boundary in the magnetosheath of comets, Venus and Mars, Adv. Space Res., 16, (4)153-(4)158, 1995.

Sauer, K., A. Bogdanov, K. Baumgärtel, and E. Dubinin, Plasma environment of comet Wirtanen during its low-activity stage, Planet. Space Sci. 44, 715-729, 1996a.

Sauer, K., A. Bogdanov, K. Baumgärtel, and E. Dubinin, Bi-ion discontinuities at weak solar wind mass-loading, Physica Scripta, T63, 111-118, $1996 b$.

Sauer, K., E. Dubinin, and K. Baumgärtel, Bow shock splitting in bi-ion flows, Geophys. Res. Lett., 23, 3643-3646, $1996 \mathrm{c}$.

Sauer, K., E. Dubinin, and K. Baumgärtel, Bi-ion structuring in the magnetosheath of Mars. Theoretical modelling, Adv. Space Res., 20, (2)137142, 1997.

Sauer, K., K. Baumgärtel, E. Dubinin, and V. Tarasov, Low-frequency electromagnetic waves and instabilities within the Martian bi-ion plasma, Earth Planets Space, 50, 269-278, 1998.

Trotignon, J. G., E. Dubinin, R. Grard, S. Barabash, and R. Lundin, The Martian planetopause as seen by the plasma wave system onboard Phobos-2, J. Geophys. Res., 101, 24965-24977, 1996.

K. Sauer (e-mail: sauer@linmpi.mpg.de), E. Dubinin, and K Baumgärtel 